\title{
Benazepril hydrochloride improves diabetic nephropathy and decreases proteinuria by decreasing ANGPTL-4 expression
}

\author{
Lingyu Xue*, Xiaoqing Feng, Chuanhai Wang, Xuebin Zhang, Wenqiang Sun and Kebo Yu
}

\begin{abstract}
Background: This study aimed to investigate the effects of benazepril hydrochloride (BH) on proteinuria and ANGPTL-4 expression in a diabetic nephropathy (DN) rat model.

Methods: A total of 72 Wistar male rats were randomly divided into three groups: normal control (NC), DN group and $\mathrm{BH}$ treatment $(\mathrm{BH})$ groups. The DN model was induced by streptozotocin (STZ). Weight, glucose, proteinuria, biochemical indicators and the kidney weight index were examined at 8, 12 and 16 weeks. In addition, ANGPTL-4 protein and mRNA expressions were assessed by immunohistochemistry and qRT-PCR, respectively. Relationships between ANGPTL-4 and biochemical indicators were investigated using Spearman analysis.

Results: Weight was significantly lower but glucose levels were significantly higher in both the DN and BH groups than in the NC group $(P<0.05)$. Compared with the DN group, proteinuria, urea, creatinine, triglycerides and total cholesterol levels were decreased, whereas the albumin level was increased after $\mathrm{BH}$ treatment (all $P<0.05$ ).

Furthermore, $\mathrm{BH}$ diminished kidney volume and ameliorated the pathological changes associated with DN.

ANGPTL-4 expression was significantly decreased after BH treatment, and ANGPTL-4 expression was highly correlated with biochemical indicators of DN $(P<0.05)$.
\end{abstract}

Conclusions: Benazepril hydrochloride improves DN and decreases proteinuria by decreasing ANGPTL-4 expression.

Keywords: Benazepril hydrochloride, Diabetic nephropathy, ANGPTL-4, Proteinuria

\section{Background}

Diabetic nephropathy (DN) is one of the major chronic microvascular complications of diabetes, which seriously impacts a large number of people worldwide $[1,2]$. DN incidence is increasing with the rapid growth of diabetes year by year, and $\mathrm{DN}$ has become a major cause of endstage renal disease (ESRD) [3]. According to the statistics of the International Diabetes Federation (IDF), the number of diabetic patients has reached 37 million worldwide, with about 4.6 million of them dying in 2011. Furthermore, rapid growth in the incidence of diabetes is occurring in China and other developing countries, leading to a heavy social and economic burden. $\mathrm{DN}$ has become the first major disease requiring dialysis treatment, with expensive associated costs [4].

\footnotetext{
* Correspondence: xuely2539@163.com

Department of Nephrology, the Affiliated Hospital of Taishan Medical University, No. 706 Taishan Street, Taian, Shandong Province 271000, China
}

Microalbuminuria is generally the first clinical manifestation of $\mathrm{DN}$, and it further promotes clinical progression. Without effective intervention, proteinuria levels further increase and eventually leads to kidney failure. Proteinuria is not only a marker for DN severity but is also closely associated with $\mathrm{DN}$ progression.

A recent study has found that vascular endothelial dysfunction is important for diabetic vascular disease progression [5]. Multiple angiopoietin-related genes, such as Ang1, Ang2, ANGPTL-1, ANGPTL-2 and ANGPTL-3, are critical for angiogenesis $[6,7]$. ANGPTL-4 is homologous with Ang2 at the amino acid level, suggesting that ANGPTL-4 may be associated with angiogenesis. ANGPTL-4 overexpression in rats induce and increase nephrotic-range proteinuria, whereas proteinuria is markedly lower in ANGPTL-4 knock-in mice [8]. Increasing evidence has shown that ANGPTL-4 is overexpressed in minimal change nephropathy, which is 
correlated with proteinuria $[9,10]$. Benazepril hydrochloride $(\mathrm{BH})$ is a commonly used drug for the clinical treatment of DN, which not only decreases blood pressure but also decreases the proteinuria level [11]. Although several studies have investigated how $\mathrm{BH}$ improves $\mathrm{DN}$, the underlying mechanism is still far from completely clear and the effect of DN on ANGPTL-4 remains to be elucidated.

In the present study, we aimed to investigate the relationship between ANGPTL-4 and biochemical indices as well as proteinuria. The effects of $\mathrm{BH}$ on biochemical indices, proteinuria and ANGPTL-4 expression were also assessed. Our study provides further information on the molecular mechanism of $\mathrm{BH}$ and supplies new insights for drug development of DN by targeting ANGPTL-4.

\section{Methods}

\section{Ethics statement}

Animals were treated strictly in accordance with all animal care and experimental procedures according to the Care and Use of Laboratory Animals. All animal experiments were permitted and approved by the Committee of our hospital and all efforts were made to minimise discomfort and pain of rats.

\section{Animals and grouping}

A total of 90 healthy Wistar male rats, aged 6-8 weeks, weighing 170-200 g, were purchased from Jining Lukang Pharmaceutical Co., Ltd. (Shandong, China). All the rats were housed in a specific pathogen-free animal room at a temperature of $25{ }^{\circ} \mathrm{C} \pm 2{ }^{\circ} \mathrm{C}$, a relative humidity of $25 \%$ $30 \%$ and a standard 12-h light/dark cycle. The rats had free access to sterilised water and food. After an adaptive feeding period of 7 days, blood glucose test strips were applied to screen out ineligible rats with a fasting plasma glucose of $>7.0 \mathrm{mmol} / \mathrm{L}$ and a urine protein score $\geq 1$. Seventy-two eligible rats were randomly assigned into three groups according to a random number table, namely a normal control group (NC group, $n=24$ ), a DN group, $\mathrm{n}=24$ ) and a $\mathrm{BH}$-treated group (BH group, $\mathrm{n}=24$ ). At the same time, 12 additional rats were randomly divided into $\mathrm{DN}$ and $\mathrm{BH}$ groups for a synchronisation test to avoid excess mortality in the experiments, which might influence the statistical outcome.

\section{DN rat model}

Rats were fasted for $12 \mathrm{~h}$ before diabetes was induced in $\mathrm{DN}$ and $\mathrm{BH}$ rats with an intraperitoneal injection of $1 \%$ streptozotocin (STZ) at a dose of $60 \mathrm{mg} / \mathrm{kg}$ body weight. The rats in the $\mathrm{NC}$ group received an equal volume of saline. Blood from the tail vein was used to detect plasma glucose by an ACCU-CHEK blood glucose monitoring device (Roche) after 3 days. Development of the diabetes model was successful if the plasma glucose was
$>16.7 \mathrm{mmol} / \mathrm{L}$. Three weeks later, urinary protein levels in urine collected over $24 \mathrm{~h}$ were assessed for each rat. Urinary protein $\geq 30 \mathrm{mg} / 24 \mathrm{~h}$ indicated successful development of $\mathrm{DN}$ in the rat model. The rats in the $\mathrm{BH}$ group were then gavage fed $\mathrm{BH}$ (Lotensin, Beijing Novartis Pharma Ltd., Beijing, China) at a dose of $10 \mathrm{mg} / \mathrm{kg} / \mathrm{d}$, and the rats in the $\mathrm{NC}$ and $\mathrm{DN}$ groups were fed with an equal volume of saline. Rats were closely monitored during the whole experiment, including weight, diet, drinking, spirit and colour, etc.

\section{Sample collection and serum parameter measurements}

Blood samples were obtained by tail venepuncture to examine the glucose levels. Blood samples taken from the heart of anaesthetised rats were immediately analysed to measure serum levels of urea, creatinine, triglycerides, total cholesterol and albumin using an automatic biochemistry analyser (AU5800, Beckman Coulter Inc., Brea CA, USA). Urine was collected for 1 day before sacrifice to detect proteinuria using the nephelometry method (Siemens BN II, Deerfield, IL, USA).

\section{Histological observation}

At weeks 4, 8 and 12 after $\mathrm{BH}$ induction, eight rats from each group were sacrificed under anaesthesia to obtain the kidneys, which were fixed with $10 \%$ formalin for $24 \mathrm{~h}$ and dehydrated with graded ethanol. After transparency, the specimens were embedded into paraffin and cut into $3-\mu \mathrm{m}$ sections. The sections were then dewaxed, rehydrated and stained with haematoxylin and eosin (H\&E) for observation under an inverted microscope (Nikon YS100, Tokyo, Japan).

\section{Immunohistochemistry}

After dewaxing, rehydration and antigen retrieval, the sections were blocked with goat serum for $10-15 \mathrm{~min}$, followed by incubation with specific primary antibody at $37{ }^{\circ} \mathrm{C}$ for $2 \mathrm{~h}$. Then the sections were washed with phosphate-buffered saline (PBS) three times and incubated with biotin-labelled goat anti-rabbit IgG at $37^{\circ} \mathrm{C}$ for $15 \mathrm{~min}$. Subsequently, the sections were incubated with horseradish peroxidase (HRP)-labelled streptavidin, stained with 3,3'-diaminobenzidine and re-stained with haematoxylin. A microscope (Olympus, Japan) was used to observe staining and five random images were taken for each section. In addition, Image-Pro Plus software 6.0 (Media Cybernetics, Silver Spring, MD, USA) was used for image analysis, and the average optical density value was calculated to reflect the expression level of the proteins.

\section{Quantitative real-time PCR}

Rat kidney samples ( 100 mg) were used for extraction of total RNA by TRIzol reagent (Tiangen, Beijing, China). A reverse transcription kit (Tiangen) was used 
Table 1 The weights (g) of rats in the different experimental groups ( $n=8$ for each group at each time point)

\begin{tabular}{llll}
\hline Time (weeks) & NC group & DN group & BH group \\
\hline 8 & $375.90 \pm 25.072$ & $194.30 \pm 13.684^{*}$ & $212.98 \pm 15.187^{* \#}$ \\
12 & $503.33 \pm 27.830$ & $181.33 \pm 12.055^{*}$ & $224.78 \pm 13.826^{* \#}$ \\
16 & $538.50 \pm 28.116$ & $187.00 \pm 12.247^{*}$ & $269.93 \pm 18.291^{* \#}$ \\
\hline
\end{tabular}

$N C$ normal controls, $D N$ diabetic nephropathy, $B H$ benazepril treated group The difference comparisons were performed by one-way analysis of variance followed by multiple comparisons with the LSD test. ${ }^{*} P<0.05$ vs. NC,

${ }^{\#} P<0.05$ vs. DN to obtain cDNA. The primer sequences for real-time PCR were as follows: Angptl-4 primers 5'-TCT CAC TTC TCG CCT ACC AG-3' and 5'-CCC TAT CTC CAG TCG GTC AA-3', GAPDH primers 5'-TTC TAG AGA CAG CCG CAT CT-3' and 5'-TGG TAA CCA GGT GTC CGA TA-3'. For real-time PCR, a $2 \times$ SYBR Green PCR Master Mix kit (Applied Biosystems, Warrington, UK) was used according to the manufacturer's instructions, and amplified with a BioRad machine (Singapore). Amplification conditions were set as $94{ }^{\circ} \mathrm{C}$ for $15 \mathrm{~min}$; 40 cycles of $94{ }^{\circ} \mathrm{C}$ for $20 \mathrm{~s}, 58{ }^{\circ} \mathrm{C}$ for $30 \mathrm{~s}$, $68{ }^{\circ} \mathrm{C}$ for $30 \mathrm{~s}$; then $68{ }^{\circ} \mathrm{C}$ for $10 \mathrm{~min}$. The mRNA level

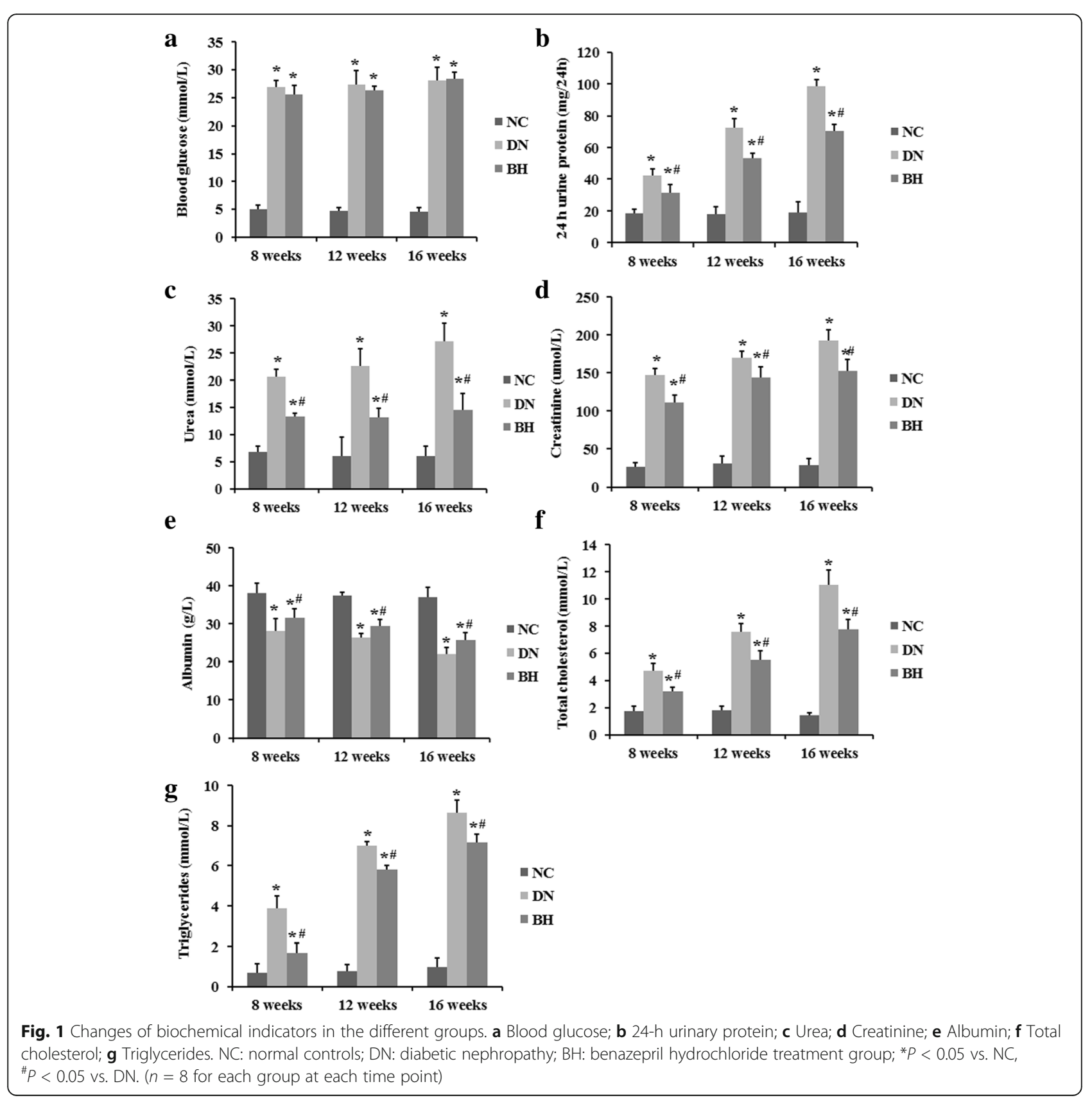


Table 2 Kidney weight index $(\mathrm{mg} / \mathrm{g})$ in different groups $(\mathrm{n}=8$ for each group at each time point)

\begin{tabular}{llll}
\hline Time (weeks) & NC group & DN group & BH group \\
\hline 8 & $3.158 \pm 0.505$ & $7.059 \pm 0.586^{*}$ & $5.894 \pm 0.418^{* \#}$ \\
12 & $3.273 \pm 0.520$ & $8.024 \pm 0.693^{*}$ & $6.103 \pm 0.521^{{ }_{*}^{*}}$ \\
16 & $3.281 \pm 0.299$ & $9.211 \pm 0.453^{*}$ & $6.723 \pm 0.283^{* \#}$ \\
\hline
\end{tabular}

$N C$ normal controls, $D N$ diabetic nephropathy, $B H$ benazepril treated group The difference comparisons were performed by one-way analysis of variance followed by multiple comparisons with the LSD test. ${ }^{*} P<0.05$ vs. NC, ${ }^{\#} P<0.05$ vs. DN

of Angptl-4 was normalised to GAPDH and the results were analysed using the $2^{-\Delta \Delta \mathrm{Ct}}$ method.

\section{Statistical analysis}

All data were expressed as mean \pm standard deviation (SD) and analysed using SPSS 19.0. The difference comparisons were performed by one-way analysis of variance followed by multiple comparisons with the LSD test. Correlation analysis between two variables was conducted using Spearman analysis. $P<0.05$ was considered as a statistically significant difference.

\section{Results}

\section{Basic characteristics of rats in the different groups}

In the NC group, rats showed normal behaviour, a shiny coat, produced normal amounts of urine without a peculiar smell, with no diarrhoea or death. Rats in the DN group showed signs of distress with less activity, body weight loss, dark and brown hair, an increased water and food intake and a larger urine volume with a peculiar smell. In addition, some rats had ring ulceration, eye lesions and diarrhoea.
After treatment with $\mathrm{BH}$, the basic characteristics of diabetic rats were notably improved. During the entire experiment, the number of dead rats was zero, five and two in the $\mathrm{NC}$, $\mathrm{DN}$ and $\mathrm{BH}$ groups, respectively. The weights of the rats are shown in Table 1. Compared with the NC group, the weights were significantly decreased in both the $\mathrm{DN}$ and $\mathrm{BH}$ groups throughout the whole experiment $(P<0.05)$. Significant differences were also found between the $\mathrm{DN}$ and $\mathrm{BH}$ groups at 16 weeks $(P<0.05)$, but not at 8 and 12 weeks $(P>0.05)$. The weight of rats in the NC group kept increasing $(P<0.05)$, whereas no obvious changes were found in the $\mathrm{DN}$ and $\mathrm{BH}$ groups $(P>0.05)$.

\section{Changes of biochemical indicators}

Glucose, proteinuria, urea, creatinine, triglycerides, total cholesterol and albumin levels are shown in Fig. 1. The results show that after treatment with STZ, the glucose levels in both the $\mathrm{DN}(26.910 \pm 1.115)$ and $\mathrm{BH}(25.611 \pm 1.587)$ groups were $>16.7 \mathrm{mmol} / \mathrm{L}$ at week 8 , and the level of $24-\mathrm{h}$ proteinuria in the $\mathrm{DN}(42.525 \pm 4.157)$ and $\mathrm{BH}$ (31.281 \pm 5.123$)$ groups were both $>30 \mathrm{mg} / 24 \mathrm{~h}$ at week 8 , which suggested that the rats were diabetic and that the DN rat models were successfully constructed. Glucose and proteinuria levels were significantly higher in the DN and $\mathrm{BH}$ groups than in the $\mathrm{NC}$ group (Fig. $1 \mathrm{a}$ and $\mathrm{b}, P<0.05$ ). After $\mathrm{BH}$ treatment, proteinuria levels significantly decreased compared with the DN group, although no significant difference in glucose levels was found between these two groups. In accordance with the marked reduction in proteinuria, urea, creatinine, triglycerides and total cholesterol levels were also decreased, whereas albumin levels were increased after $\mathrm{BH}$ treatment (Fig. $1 \mathrm{c}-\mathrm{g}, P<0.05$ ).

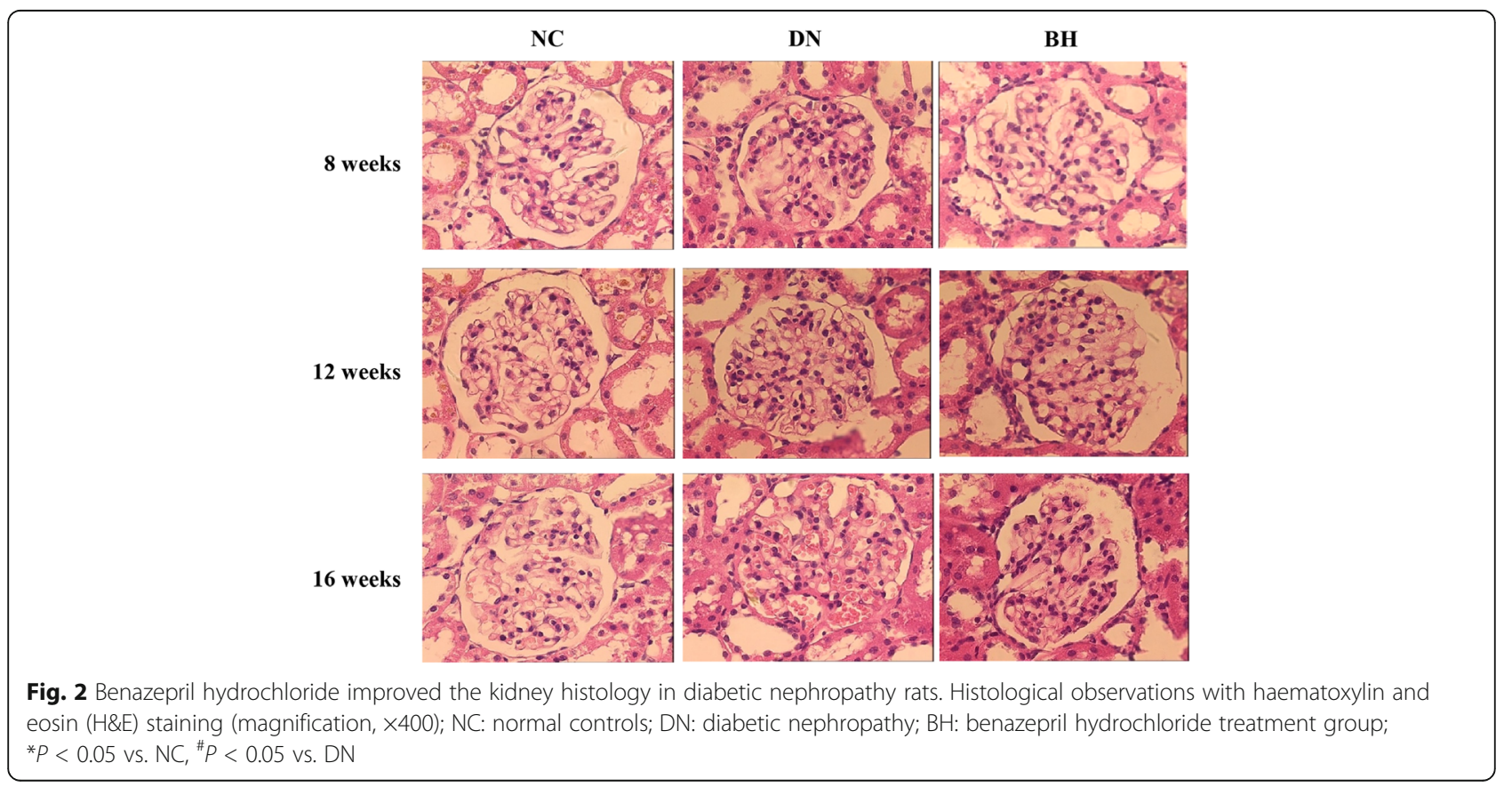



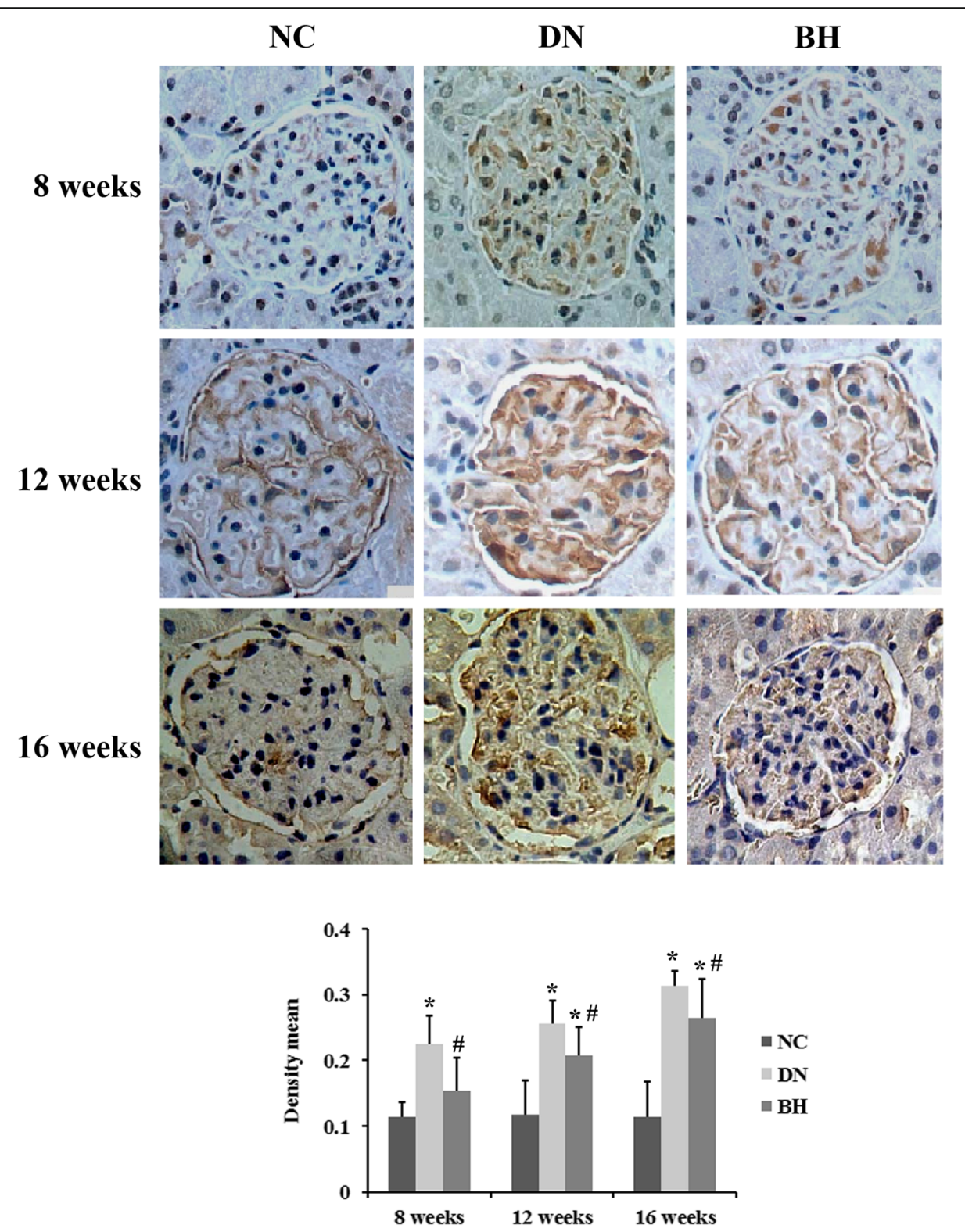

Fig. 3 Benazepril hydrochloride decreased ANGPTL-4 expression in diabetic nephropathy rat kidneys. ANGPTL-4 protein levels in different groups examined by immunohistochemistry (magnification, $\times 400$ ); NC: normal controls; DN: diabetic nephropathy; BH: benazepril hydrochloride treatment group; ${ }^{*} P<0.05$ vs. NC, ${ }^{\#} P<0.05$ vs. DN ( $n=8$ for each group at each time point)

$\mathrm{BH}$ treatment diminished volume and the infiltration of inflammatory cells in DN rat kidneys

We used the kidney weight index (weight of unilateral kidney weight / body weight) to represent the increase in kidney volume (Table 2). As a result, increased kidney volume was found in the model (DN and $\mathrm{BH})$ groups $(P<0.05)$ and $\mathrm{BH}$ treatment significantly decreased the kidney weight index compared with the DN group $(P<0.05)$.

Rats in the NC group had normal glomerular volume, shape, and structure (Fig. 2). For the DN group, the kidneys had bigger glomeruli, a thickened glomerular basement membrane, larger mesangial cells and a large amount of inflammatory cell infiltration. $\mathrm{BH}$ treatment markedly improved the pathological conditions, decreasing the mesangial cell size and matrix thickness, as well as dampening the infiltration of inflammatory cells (Fig. 2).

\section{$\mathrm{BH}$ treatment decreased ANGPTL-4 expression in DN rat kidneys}

We noted that ANGPTL-4 expression was upregulated in the DN group at weeks 8,12 and 16 compared with that in the NC group, as determined by 


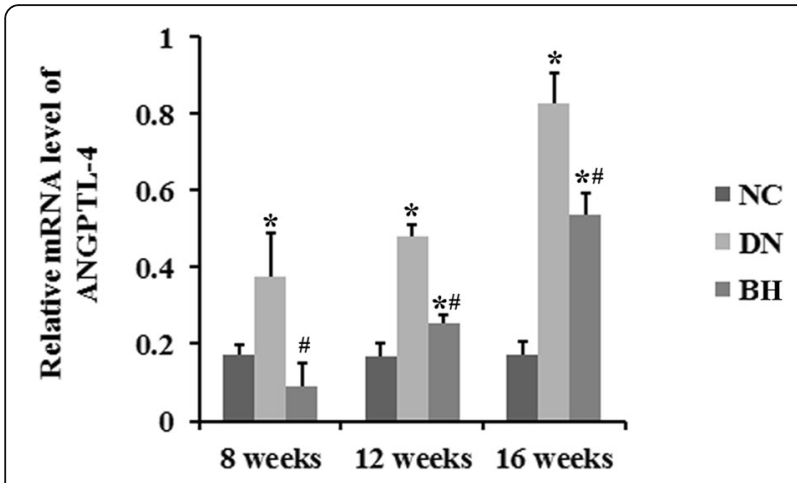

Fig. 4 Benazepril hydrochloride decreased the mRNA level of ANGPTL-4 in diabetic nephropathy rat kidneys. NC: normal controls; DN: diabetic nephropathy; $\mathrm{BH}$ : benazepril hydrochloride treatment group; ${ }^{*} P<0.05$ vs. $N C,{ }^{\#} P<0.05$ vs. DN ( $n=8$ for each group at each time point)

immunohistochemistry $(P<0.05$, Fig. 3$)$. This finding was also confirmed at the mRNA level by qRT-PCR (Fig. 4). Further, an increase of ANGPTL-4 expression was found in the $\mathrm{DN}$ and $\mathrm{BH}$ groups at weeks 12 and 16 compared with that at week $8(P<0.05)$. Compared with the DN group, protein and mRNA levels of ANGPTL-4 were significantly decreased after treatment with $\mathrm{BH}$ $(P<0.05)$.

\section{The ANGPTL-4 level was significantly correlated with the biochemical indicators of DN}

The correlations between ANGPTL-4 level and 24-h urine protein, total cholesterol, triglycerides, creatinine and kidney weight index were also analysed (Table 3). As a result, ANGPTL-4 levels positively correlated with the amount of 24-h urine protein, creatinine and the kidney weight index $(r=0.907, P<0.01 ; r=0.817$, $P<0.01 ; r=0.882, P<0.01$; respectively), whereas there was a negative correlation between the ANGPTL-4 level and total cholesterol $(r=0.536, P<0.01)$.

\section{Discussion}

In this study, we showed that glomerular size and ANGPTL-4 expression were significantly increased, which most likely associated with the heavy proteinuria in DN rats. Furthermore, the ANGPTL-4 expression level significantly correlated with the amounts of $24-\mathrm{h}$ urine protein and creatinine, the kidney weight index and total cholesterol levels. However, BH treatment markedly decreased ANGPTL-4 expression, which was accompanied by a reduction of proteinuria and an improved glomerular pathology in the DN rats.

An activated renin-angiotensin-aldosterone system is one of the most important contributors to DN pathogenesis [12]. Angiotensin-converting enzyme inhibitor (ACEI) drugs have been used for the treatment of STZinduced diabetic rats and they decrease the expression of growth factor receptors and inhibit signalling [13]. Benazepril is a commonly used ACEI drug that has been used for the treatment of hypertension and heart failure $[14,15]$. Recently, benazepril has been shown to generate a strong flow-mediated vasodilation response and a remarkable reduction in $\mathrm{C}$-reactive protein in diabetic patients [16]. Meanwhile, $\mathrm{BH}$ has been widely used for renal diseases, such as renal hypertension, renal failure and DN $[17,18]$. According to our results, $\mathrm{BH}$ notably improved the morphology of glomeruli and decreased the proteinuria in $\mathrm{DN}$ rats, suggesting that $\mathrm{BH}$ was an effective treatment for $\mathrm{DN}$ in the rat model.

ANGPTL-4 is involved in wound healing, cancer, angiogenesis and redox regulation, as well as lipid and glucose metabolism. Initial studies have revealed increased podocyte expression of ANGPTL-4 in minimal change disease (MCD), and it causes increased proteinuria in membranous nephropathy $(\mathrm{MN})[8$, 19]. Consistent with the previous studies, ANGPTL-4 was also upregulated in DN rats in our study. Downregulating ANGPTL-4 expression or promoting the conversion of ANGPTL- 4 has been shown to decrease proteinuria in rats with $\mathrm{MCD}$ and $\mathrm{DN}[8,20]$. Considering ANGPTL-4 is a major molecular mediator of nephrotic syndrome, it has been identified as an ideal candidate for the treatment of proteinuric disorders caused by chronic kidney disease [20]. ManNAc decreases proteinuria in MCD rats, accompanied by a decrease of ANGPTL-4 expression [8]. Tacrolimus is a calcineurin inhibitor and it can decrease proteinuria in MN by targeting ANGPTL-4 [21]. In this study, ANGPTL-4 expression was also significantly lower after $\mathrm{BH}$ treatment compared with the DN model group, which indicates that ANGPTL-4 might be involved in the treatment effects of $\mathrm{BH}$ on DN.

An increasing number of reports have described the involvement of cytokines in the occurrence and progression of DN $[22,23]$, and the infiltration of inflammatory cells was also found in our H\&E analyses. Multiple

Table 3 The correlations between ANGPTL-4 expression and biochemical indicators

\begin{tabular}{|c|c|c|c|c|c|c|c|}
\hline & & 24-h urine protein & Albumin & Creatinine & Triglycerides & Total cholesterol & Kidney weight index \\
\hline \multirow[t]{2}{*}{ ANGPTL-4 } & $r$ & 0.907 & -0.226 & 0.817 & 0.219 & 0.536 & 0.882 \\
\hline & $p$ & 0.000 & 0.160 & 0.000 & 0.174 & 0.000 & 0.000 \\
\hline
\end{tabular}

Correlation analysis between two variables was conducted using Spearman analysis; $r$ is the correlation coefficient and $P<005$ was considered as significantly correlated 
studies have suggested that ANGPTL-4 is involved in inflammatory-related processes [24, 25]. Therefore, the improved inflammation in the $\mathrm{BH}$ group might be related to the low expression of ANGPTL-4. After $\mathrm{BH}$ treatment, DN-related biochemical indicators were returned to normal levels, to varying degrees. Urea, creatinine, triglycerides and total cholesterol levels were decreased, whereas the albumin level was increased compared with the $\mathrm{DN}$ group. Additionally, the ANGPTL-4 level positively correlated with the amount of 24-h urine protein, creatinine and the kidney weight index, and negatively correlated with total cholesterol according to Spearman analysis. Thus, we conclude that ANGPTL-4 might be associated with the regulation of these biochemical indicators by $\mathrm{BH}$.

However, this study also has some limitations. A previous study showed that STZ could not only induce DN but also hypertension [26]. Because this study was focused on the effects of $\mathrm{BH}$ on $\mathrm{DN}$, blood pressure was not measured and the influence of hypertension on ANGPTL-4 was not considered. Therefore, the effect of hypertension on ANGPTL-4 should be investigated in further studies. Despite this, the present study also demonstrated that $\mathrm{BH}$ can decrease proteinuria and improve $\mathrm{DN}$, which might be associated with ANGPTL-4. In addition, this study revealed that ANGPTL-4 might be associated with the treatment effects of $\mathrm{BH}$ on $\mathrm{DN}$. However, whether $\mathrm{BH}$ improved $\mathrm{DN}$ by targeting ANGPTL-4 is still unclear and a further study focused on the overexpression or deficiency of ANGPTL-4 should be performed to investigate a possible causal relationship between ANGPTL-4 expression and the effects of $\mathrm{BH}$ on improvement of $\mathrm{DN}$.

\section{Conclusions}

In conclusion, this study demonstrated that $\mathrm{BH}$ decreased proteinuria and improve $\mathrm{DN}$, which might be closely associated with ANGPTL-4. However, the specific mechanism is not fully understood and needs to be examined in further studies.

\section{Abbreviations}

ACEI: Angiotensin-converting enzyme inhibitor; BH: Benazepril hydrochloride; DBA: Diaminobenzidine; DN: Diabetic nephropathy; ESRD: End-stage renal disease; H\&E: Haematoxylin and eosin; IDF: International Diabetes Federation; MCD: Minimal change disease; PBS: Phosphate-buffered saline; SD: Standard deviation

\section{Acknowledgements \\ None.}

\section{Funding}

None.

\section{Availability of data and materials}

The datasets generated during the current study are not publicly available due to a following mechanism research based on these data.

\section{Authors' contributions}

LX carried out the molecular studies, XF participated in the sequence alignment, LX, XF and CW drafted the manuscript. XZ carried out the study. WS participated in the sequence alignment. CW and KY participated in the design of the study and performed the statistical analysis. WS conceived of the study, and participated in its design and coordination and helped to draft the manuscript. All authors read and approved the final manuscript.

\section{Ethics approval and consent to participate}

Animals were treated strictly in accordance with all animal care and experimental procedures according to the Care and Use of Laboratory Animals. All animal experiments were permitted and approved by the Committee of our hospital and all efforts were made to minimise discomfort and pain of rats.

\section{Consent for publication}

Not applicable.

\section{Competing interests}

The authors declare that they have no competing interests.

\section{Publisher's Note}

Springer Nature remains neutral with regard to jurisdictional claims in published maps and institutional affiliations.

Received: 20 September 2015 Accepted: 22 September 2017 Published online: 04 October 2017

\section{References}

1. Matsui T, Yamagishi S, Takeuchi M, Ueda S, Fukami K, Okuda S. Nifedipine inhibits advanced glycation end products (AGEs) and their receptor (RAGE) interaction-mediated proximal tubular cell injury via peroxisome proliferator-activated receptor-gamma activation. Biochem Biophys Res Commun. 2010;398(2):326-30.

2. Kong LL, Wu H, Cui WP, Zhou WH, Luo P, Sun J, Yuan H, Miao LN. Advances in murine models of diabetic nephropathy. J Diabetes Res. 2013;2013:797548.

3. Keane WF, Brenner BM, De Zeeuw D, Grunfeld J-P, Mcgill J, Mitch WE, Ribeiro AB, Shahinfar S, Simpson RL, Snapinn SM. The risk of developing end-stage renal disease in patients with type 2 diabetes and nephropathy: the RENAAL study. Kidney Int. 2003;63(4):1499-507.

4. Dronavalli S, Duka I, Bakris GL. The pathogenesis of diabetic nephropathy. Nat Clin Pract Endocrinol Metab. 2008:4(8):444-52.

5. Yamamoto $Y$, Maeshima $Y$, Kitayama $H$, Kitamura S, Takazawa $Y$, Sugiyama $H_{\text {, }}$ Yamasaki Y, Makino H. Tumstatin peptide, an inhibitor of angiogenesis, prevents glomerular hypertrophy in the early stage of diabetic nephropathy. Diabetes. 2004:53(7):1831-40.

6. Salchell S, Mathieson PW. Angiopoietins: microvascular modulators with potential roles in glomerular pathophysiology. J Nephrol. 2003;16(2):168-78.

7. Lai KN, Leung JC, Tang SC. The renin-angiotensin system. Contrib Nephrol. 2011;170:135-44.

8. Clement LC, Avila-Casado C, Mace C, Soria E, Bakker WW, Kersten S, Chugh SS. Podocyte-secreted angiopoietin-like-4 mediates proteinuria in glucocorticoid-sensitive nephrotic syndrome. Nat Med. 2011;17(1):117-22.

9. Reiser J. Filtering new facts about kidney disease. Nat Med. 2011;17(1):44-5.

10. Chugh SS, Clement LC, Macé C. New insights into human minimal change disease: lessons from animal models. Am J Kidney Dis. 2012;59(2):284-92.

11. Liu X, Liu L, Chen P, Zhou L, Zhang Y, Wu Y, Jiang L, Cheng D, Huang W, Yi D. Clinical trials of traditional Chinese medicine in the treatment of diabetic nephropathy —A systematic review based on a subgroup analysis. J Ethnopharmacol. 2014;151(2):810-9.

12. Cooper ME. Interaction of metabolic and haemodynamic factors in mediating experimental diabetic nephropathy. Diabetologia. 2001;44(11):1957-72.

13. Allard J, Buleon M, Cellier E, Renaud I, Pecher C, Praddaude F, Conti M, Tack I, Girolami JP. ACE inhibitor reduces growth factor receptor expression and signaling but also albuminuria through B2-kinin glomerular receptor activation in diabetic rats. Am J Physiol Renal Physiol. 2007;293(4):F1083-92.

14. Naguib IA, Abdelaleem EA, Draz ME, Zaazaa HE. Linear support vector regression and partial least squares chemometric models for determination of Hydrochlorothiazide and Benazepril hydrochloride in presence of related impurities: a comparative study. Spectrochim Acta A Mol Biomol Spectrosc. 2014;130:350-6. 
15. Brittain HG. Profiles of Drug Substances, Excipients, and Related Methodology. Analy Profiles Drug Subst Excipients. 2002;29:1-5

16. Gismondi RA, Oigman W, Bedirian R, Pozzobon CR, Ladeira MC, Neves MF. Comparison of benazepril and losartan on endothelial function and vascular stiffness in patients with Type 2 diabetes mellitus and hypertension: A randomized controlled trial. JRAAS. 2015;16:967.

17. Zhi-hui B, Zhen M. Combination Therapy of Benazepril Hydrochloride with Hydrochlor-othiazide for Renal Hypertension. Guide China Med. 2012;34:321.

18. Wang W, Li L, Zhou Z, Gao J, Sun Y. Effect of spironolactone combined with angiotensin-converting enzyme inhibitors and/or angiotensin II receptor blockers on chronic glomerular disease. Exp Ther Med. 2013;6(6):1527-31.

19. Clement LC, Mace C, Avila-Casado C, Joles JA, Kersten S, Chugh SS. Circulating angiopoietin-like 4 links proteinuria with hypertriglyceridemia in nephrotic syndrome. Nat Med. 2014;20(1):37-46.

20. Chugh SS, Mace C, Clement LC, Del Nogal AM, Marshall CB. Angiopoietinlike 4 based therapeutics for proteinuria and kidney disease. Front Pharmacol. 2014;5:23.

21. Peng L, Ma J, Cui R, Chen X, Wei SY, Wei QJ, Li B. The calcineurin inhibitor tacrolimus reduces proteinuria in membranous nephropathy accompanied by a decrease in angiopoietin-like-4. PLoS One. 2014;9(8):e106164.

22. Schnaper HW, Hayashida T, Hubchak SC, Poncelet AC. TGF-beta signal transduction and mesangial cell fibrogenesis. Am J Physiol Renal Physiol. 2003;284(2):F243-52.

23. Flyvbjerg A. Putative pathophysiological role of growth factors and cytokines in experimental diabetic kidney disease. Diabetologia. 2000;43(10):1205-23.

24. Quintero P, González-Muniesa P. Garcia-Diaz DF, Martínez JA. Effects of hyperoxia exposure on metabolic markers and gene expression in 3T3-L1 adipocytes. J Physiol Biochem. 2012;68(4):663-9.

25. Oike Y, Tabata M. Angiopoietin-Like Proteins Potential Therapeutic Targets for Metabolic Syndrome and Cardiovascular Disease. Circ J. 2009;73(12):2192-7.

26. Musial DC, da Silva Júnior ED, da Silva RM, Miranda-Ferreira R, LimaLandman MTR, Jurkiewicz A, García AG, Jurkiewicz NH. Increase of angiotensin-converting enzyme activity and peripheral sympathetic dysfunction could contribute to hypertension development in streptozotocin-induced diabetic rats. Diab Vasc Dis Res. 2013;10:498, 1479164113496441

\section{Submit your next manuscript to BioMed Central and we will help you at every step:}

- We accept pre-submission inquiries

- Our selector tool helps you to find the most relevant journal

- We provide round the clock customer support

- Convenient online submission

- Thorough peer review

- Inclusion in PubMed and all major indexing services

- Maximum visibility for your research

Submit your manuscript at www biomedcentral.com/submit

) Biomed Central 